\title{
Prática de biblioterapia no Brasil e no exterior: principais experiências com a terapia pela leitura
}

\author{
Bibliotherapy practice in Brazil and abroad: some experiences with \\ reading therapy
}

\section{Práctica de la biblioterapia en Brasil y en el extranjero: algunas experiencias con la terapia de lectura}

\author{
Andréa Pereira dos Santos ${ }^{1, a}$ \\ andreabiblio@ufg.br | https://orcid.org/o0oo-0001-5410-5500
}

Natália Rocha ${ }^{1, b}$

rochanatttalia@gmail.com | https://orcid.org/0000-0002-7900-8333

Larissa Andrade Batista Cavalcanti, ${ }^{i, c}$

larissa.andrade@discente.ufg.br | https://orcid.org/0000-0003-0600-6748

${ }^{1}$ Universidade Federal de Goiás, Faculdade de Informação e Comunicação. Goiânia, GO, Brasil.

a Doutorado em Geografia pela Universidade Federal de Goiás.

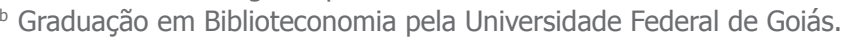

c Especialista em Letramento Informacional pela Universidade Federal de Goiás.

\section{RESUMO}

O presente estudo tem como objetivo conhecer as principais experiências com a biblioterapia voltadas para pessoas acometidas por doenças variadas, sejam físicas e/ou mentais, ou sociais no Brasil e no exterior. Justifica-se por apresentar a função terapêutica da leitura. Sua base são os conceitos teóricos e históricos da biblioterapia, o conhecimento das principais experiências com a biblioterapia no Brasil e um comparativo entre a realidade nacional e internacional da sua utilização. Para tanto, foi feita uma pesquisa exploratória, qualitativa e bibliográfica em bases de dados nacionais e internacional (LISA) com material publicado sobre experiências que utilizassem a biblioterapia para o bem-estar do indivíduo. Desse modo, observa-se que o Brasil caminha para ampliação da utilização da terapia, enquanto outros países a utilizam nos âmbitos hospitalar, escolar, prisional, entre outros. Isso permite concluir que a biblioterapia proporciona saúde e bem-estar aos que dela fazem uso.

Palavras-chave: Biblioterapia; Programas de biblioterapia; Biblioterapia com pacientes; Pacientes hospitalizados; Leitura terapêutica.

\section{ABSTRACT}

This study aims to find out the main experiences with bibliotherapy aimed at people affected by various diseases, whether physical and/or mental, or social in Brazil and abroad. It is justified by presenting the therapeutic function of reading. This study is based on the theoretical and historical concepts of bibliotherapy, the knowledge of the main experiences with bibliotherapy in Brazil and comparing it with the national 
and international reality regarding its use. To this end, it is an exploratory, qualitative and bibliographic research, which searched for published material on national and international databases (LISA) on experiences that used bibliotherapy for the well-being of the individual. Thus, it is observed that Brazil is moving towards expanding the use of therapy, while other countries use it in hospitals, schools, prisons, among others. This allows us to conclude that bibliotherapy provides health and well-being to those who use it.

Keywords: Bibliotherapy; Bibliotherapy programs; Bibliotherapy with patients; Hospitalized patients; Reader therapy.

\section{RESUMEN}

Este estudio tiene como objetivo conocer las principales experiencias con biblioterapia dirigidas a personas afectadas por diversas enfermedades, físicas y/o mentales, o sociales en Brasil y en el exterior. Se justifica presentando la función terapéutica de la lectura. Este estudio se basa en los conceptos teóricos e históricos de la biblioterapia, el conocimiento de las principales experiencias con biblioterapia en Brasil y su comparación con la realidad nacional e internacional en cuanto al uso de esta terapia. Para ello, se trata de una investigación exploratoria, cualitativa y bibliográfica, que buscó material publicado en bases de datos nacionales e internacionales (LISA) sobre experiencias que utilizaron la biblioterapia para el bienestar del individuo. Así, se observa que Brasil avanza hacia la expansión del uso de la terapia, mientras que otros países la utilizan en diversos nichos, como hospital, escuela, prisión, entre otros. Esto nos permite concluir que la biblioterapia brinda salud y bienestar a quienes la utilizan.

Palabras clave: Biblioterapia; Programas de biblioterapia; Biblioterapia con pacientes; Pacientes hospitalizados; Lectura terapéutica.

Contribuição dos autores:

Concepção e desenho do estudo: Natália Rocha, Andréa Pereira dos Santos e Larissa Andrade Batista Cavalcanti.

Aquisição, análise ou interpretação dos dados: Natália Rocha, Larissa Andrade Batista Cavalcanti, Andréa Pereira dos Santos. Redação do manuscrito: Natália Rocha, Larissa Andrade Batista Cavalcanti, Andréa Pereira dos Santos.

Revisão crítica do conteúdo intelectual: Andréa Pereira dos Santos.

Declaração de conflito de interesses: não há.

Fontes de financiamento: não houve.

Considerações éticas: não há.

Agradecimentos/Contribuições adicionais: não há.

Histórico do artigo: submetido: 6 ago. 2020 | aceito: 18 fev. 2021 | publicado: 31 ago. 2021.

Apresentação anterior: não há.

Licença CC BY-NC atribuição não comercial. Com essa licença é permitido acessar, baixar (download), copiar, imprimir, compartilhar, reutilizar e distribuir os artigos, desde que para uso não comercial e com a citação da fonte, conferindo os devidos créditos de autoria e menção à Reciis. Nesses casos, nenhuma permissão é necessária por parte dos autores ou dos editores. 


\section{INTRODUÇÃO}

A compreensão da biblioterapia em seu sentido simples assume uma técnica que se utiliza da leitura e outras atividades lúdicas como coadjuvante no tratamento de pessoas acometidas por alguma doença (FREITAS et al., 2014). É comum conectarmos o tema ao ambiente hospital, porém essa é uma prática muito mais abrangente que pode ser aplicada em ambientes como escolas, asilos e presídios. O intuito é resgatar o indivíduo - que adota nesse caso o papel de leitor - do abismo das doenças psíquicas, físicas e transtornos, proporcionando bem-estar por meio de técnicas que se apropriam da leitura direcionada como forma de reestruturação social e terapêutica.

No Brasil, essa é uma área ainda recente, que ganhou maior expressividade nos anos 2000, com os estudos de Clarice Caldin (2001), professora e pesquisadora da Universidade Federal de Santa Catarina. Isso nos instiga a buscar por experiências em centros de cuidado e recuperação que já utilizam tal prática, tanto no cenário nacional, como internacional. Dessa forma, busca-se compreender como a biblioterapia é vista e utilizada, observando-a enquanto modo de pacificar as emoções de pessoas doentes ou atingidas por algum problema de origem física ou psíquica.

A leitura, como uma experiência serena, detém grandes poderes de transformação pessoal, pode alterar o comportamento dos sujeitos e até mesmo melhorar seus relacionamentos e a sua percepção das dificuldades da vida, ajudando, assim, a terem novas perspectivas e modos de pensar.

Nesse sentido, o estudo busca apresentar a função terapêutica da leitura, que tem contribuído para a melhora da qualidade de vida dos muitos indivíduos. O poder da biblioterapia como tratamento consiste na transmissão das experiências advindas da leitura, que levam a um processo de crescimento e bem-estar.

A biblioterapia auxilia o autoconhecimento pela reflexão, reforça padrões sociais desejáveis, proporciona desenvolvimento emocional e auxilia na mudança de comportamento. Para tanto, aponta-se que a literatura ficcional é a mais indicada para garantir uma experiência emocional do leitor, efetivando a terapia de introspecção capaz de efetuar mudanças (CALDIN, 2001). Portanto, sua interpretação de mundo e conceitos podem ser transmitidos, mas os significados são pessoais e mutáveis.

Frente ao contexto apresentado, pretende-se levantar os principais programas e projetos existentes no Brasil e em outros países sobre biblioterapia voltada para pacientes acometidos por qualquer tipo de enfermidade física, psíquica ou por questões sociais. Vale destacar que a biblioterapia se divide em 3 nichos: o do crescimento, que estimula a diversão e a educação; o que tem o objetivo de informar e preparar para o tratamento hospitalar; e o imaginativo, que serve para desinibir os sentimentos e tratar os problemas emocionais (CALDIN, 2001). Assim, essa terapia aparece como um dos meios pelo qual a pessoa pode procurar apoio e conforto, e seu método ‘simples’ e direto pode ser de grande auxílio por razões inestimáveis.

Inclusive, Rodriguez-Martín, Gómez-Quintana, Díaz-Martínez e Molerio-Pérez (2013) indicam que no cenário internacional a biblioterapia avança em diversas áreas que vão muito além das experiências brasileiras, uma vez que considera o tratamento, inclusive, de problemas relacionados à nutrição.

Visto que os estudos da biblioterapia no Brasil são mais recentes, nosso problema de pesquisa buscou responder à questão: as iniciativas brasileiras para a aplicação da biblioterapia para o tratamento de doenças físicas, mentais ou problemas sociais se diferenciam das iniciativas internacionais?

O que se espera é que a prática dessa terapia no Brasil seja bem difundida entre as redes de saúde e de atenção à sociedade ao mesmo passo que em outros países, proporcionando aos pacientes os bens terapêuticos que a leitura pode agregar à sua qualidade de vida.

Posto isso, o objetivo geral da pesquisa foi conhecer as principais experiências com a biblioterapia voltadas para pessoas acometidas por doenças variadas, sejam físicas e/ou mentais, no Brasil e no exterior, e 
que contassem com a participação de bibliotecários e/outros profissionais. Quanto aos objetivos específicos, buscamos caracterizar a biblioterapia; mapear a partir do levantamento bibliográfico e documental os principais programas e/ou projetos de biblioterapia em ambientes de recuperação de saúde brasileiros e estrangeiros; e, por fim, apontar os possíveis benefícios adquiridos a partir da biblioterapia para esses pacientes.

Quantoà metodologia, trata-se de uma pesquisa exploratória, com abordagem qualitativa, fundamentação teórica baseada em levantamento bibliográfico e mapeamento dos programas e/ou projetos de biblioterapia nacionais e internacionais baseado também em levantamento bibliográfico.

\section{BIBLIOTERAPIA: CONCEITOS TEÓRICOS E HISTÓRICOS}

A biblioterapia engloba uma gama de conceitos que buscam exprimir a ideia da terapia pela leitura. A princípio, ela está ligada à sua etimologia, que deriva da junção de duas palavras de origem grega: biblion (todo tipo de material bibliográfico ou de leitura) e therapei (tratamento, cura ou restabelecimento) (RIBEIRO, 2006).

Segundo Ribeiro (2006), acredita-se que sua gênese vem do antigo Egito, apesar de não haver uma data específica, mas seu uso, a princípio, se dava pela leitura de histórias que entretinham crianças, jovens e adolescentes, procurando ocupar o tempo ocioso, até que esse recurso foi identificado como instrumento terapêutico, passando então a ser utilizado em isolamentos existentes nas prisões, hospitais e manicômios, até os dias atuais.

De acordo com os relatos de Ferreira (2008), o faraó Rammsés II colocou na frente de sua biblioteca a frase: "Remédios para alma" (ALVES, 1982 apud FERREIRA, 2003, p. 36). As bibliotecas eram instaladas em templos denominados "casas de vida" (MONTET, 1989 apud FERREIRA, 2003, p. 36) e eram conhecidas como locais de alto respeito, sabedoria e espiritualidade. A autora, que também cita outros locais, conta que os gregos também associaram os livros a uma forma de tratamento médico e espiritual ao conceberem suas bibliotecas como "a medicina da alma" (MARCINKO, 1989 apud FERREIRA, 2003, p. 36). Portanto, é fácil perceber que, desde muito cedo na história, as vantagens de ser um leitor já eram percebidas e apreciadas. Com o passar do tempo, essa prática foi naturalizada entre as nações, tornando-se cada vez mais comum.

Para Ouaknin (1996, p. 198), a biblioterapia "é primariamente uma filosofia existencial e uma filosofia do livro", que diz que o homem é um "ser dotado de uma relação com o livro". Olhando por essa esfera, o ato de ler permite ao homem compreender o texto e se compreender, se enxergar por meio da leitura, fazer parte daquilo que lê, abrir a mente para coisas novas, ideias diferenciadas. Como diz o autor, "as variações imaginativas que a literatura opera sobre o real" (p. 200).

A biblioterapia, portanto, propõe práticas de leitura que proporcionem a interpretação dos textos. Mesmo que já se falasse de práticas e técnicas que utilizassem a leitura dirigida, o termo Biblioterapia somente foi colocado em um dicionário médico em 1941, no Dorland’s Illustrated Medical Dictionary, como "emprego de livros e de sua leitura no tratamento de doenças mentais" (ALVES, 1982, p. 55). Ainda que o termo 'biblioterapia' pareça relacionar-se a 'terapia', na verdade, envolve muito mais do que a cura dos sintomas dos indivíduos, age como um despertador para atitudes.

Bueno e Caldin (2005) citam Faria Filho (1998, p. 54) ao afirmar que "O livro é a forma mais rica de se obter conhecimento, com um poder incomparável de penetração e irradiação”. O método biblioterapêutico é, portanto, uma ativação do eu, do ser em potencial que existe em seu interior e que pode trazer dinamismo por meio da linguagem. A linguagem conduz o homem para além do que ele é; as palavras não são nulas, o potencial dele se maximiza, se libertando na mente e depois para fora, por meio de ações.

Peça fundamental para a biblioterapia, a leitura pode ser definida como um ato individual e voluntário que capacita cada um individualmente a ter novas experiências com a informação transformando-a em 
conhecimento. Chartier (1996, p. 77) diz que "a leitura é sempre apropriação, invenção, produção de significados”. É uma experiência que deve ser vivida no cotidiano e se torna pessoal para cada indivíduo. Por meio da leitura é possível compreender aspectos além daqueles que já nos são próprios, estendendo, assim, o conhecimento.

O texto existe porque há um leitor para dar significado, uma voz, uma entonação diferente. Todos aqueles que leem interpretam de maneiras diferentes. Assim, para cada grupo de leitores existem muitas maneiras de ler e extrair conhecimentos diferentes.

Ao contextualizar a origem da biblioterapia, encontramos percepções que vão além da leitura propriamente dita de uma obra literária. Portanto, não se pode resumir a biblioterapia apenas a uma terapia baseada no livro, ela supera essa perspectiva ao ser percebida como o cuidado com o outro por meio das histórias, sejam elas lidas, narradas ou dramatizadas. Esse cuidado também se dá por meio do diálogo e da interação com todos os envolvidos no processo para que seja possível a produção de sentido e a construção de um novo texto e de um novo eu, o que ocorre a partir da interpretação e da compreensão (CHARTIER, 1999).

Nesse sentido, o livro é um instrumento que traz a essência para a prática biblioterapêutica, mas não somente ele faz o 'milagre', é uma questão de entrega à narrativa e interpretação da história, e essa sim proporciona o bem-estar ao indivíduo.

Discutido o caráter significativo e a essência da biblioterapia, faz-se necessário compreender também seu conceito prático. Esse diz respeito a uma atividade pré-planejada que envolve elementos de leitura e discussões conduzidas como um tratamento de auxílio à saúde sob a orientação de profissionais, objetivando alcançar pessoas com problemas comportamentais e emocionais (RIBEIRO, 2006).

Dito isso, a compreensão da biblioterapia para este estudo vai ao encontro de experiências que utilizam a biblioterapia como meio de promoção de saúde e bem-estar ao indivíduo.

\section{MATERIAIS E MÉTODOS}

O percurso metodológico deste estudo tem como base a pesquisa exploratória. Martins e Lintz (2000) a definem como uma metodologia voltada para pesquisa cujo objetivo é o estudo de uma unidade que se analisa profunda e intensamente. Busca-se descrever as ações da prática biblioterapêutica no Brasil e no exterior e conhecer o universo da biblioterapia, com suas características, os seus usos e resultados.

Pela natureza dos dados obtidos, essa é uma pesquisa qualitativa, uma vez que se preocupou com a compreensão, a interpretação do fenômeno, considerando também o significado que os outros dão às suas práticas (GONSALVES, 2007).

Quanto aos procedimentos técnicos utilizados para o levantamento de dados e informações, trata-se de uma pesquisa bibliográfica, pois é desenvolvida com base em materiais já publicados, principalmente artigos, dissertações e trabalhos de conclusão de curso.

Para o levantamento de dados nacionais, foram utilizadas as bases de dados Scielo, Biblioteca Virtual em Saúde (BVS) e Google Scholar. Quanto ao levantamento internacional, optamos pela base Library and Information Science Abstracts (LISA) por representar um recorte de experiências voltadas para as práticas na área da Biblioteconomia. Nota-se que para a pesquisa nacional optamos por um maior número de bases de dados na tentativa de fazer uma busca exaustiva das principais experiências, uma vez que os estudos no país ainda são recentes.

Quanto às palavras-chave, na pesquisa nacional foram utilizadas 'biblioterapia' e 'biblioterapia experiências'. Já na pesquisa internacional utilizou-se somente o termo ‘bibliotherapy', já que ele recuperou uma quantidade significativa de itens, mesmo refinando para palavras do título. Na busca nacional não houve definição de temporalidade para que fosse recuperada a maior quantidade de resultados, enquanto 
a internacional se deu entre 2010 e 2020 por apresentar uma quantidade relevante de resultados, sendo necessário refinar a busca.

Vale ressaltar que não se pretende neste artigo estabelecer o estado da arte do tema em língua estrangeira, já que nossa intenção foi encontrar exemplos de experiências mais relevantes ao analisar trabalhos nacionais e internacionais, verificando assim a ligação com a biblioteconomia e não com a intensão de esgotar a discussão desse assunto.

\section{PROGRAMAS DE BIBLIOTERAPIA NO BRASIL}

Ao pesquisar e expor alguns projetos de biblioterapia no Brasil, percebem-se os benefícios adquiridos por meio dessa técnica, tais como o controle das emoções, alegria e a melhora de comportamento, entre outros. É importante observar as iniciativas dos profissionais que tiveram o ímpeto de trabalhar a biblioterapia nos ambientes de recuperação e ver os modos como utilizaram a leitura em atividades lúdicas, como contação de histórias, filmes, desenhos, teatro, juntamente com um acervo variado para complementar as atividades, incentivando o gosto e a estima pela leitura. Os benefícios descritos pelos trabalhos buscados mostram que a biblioterapia é uma prática diversificada capaz de atravessar fronteiras impostas pela sociedade e que se importa com os pacientes de modo profundo, buscando alívio tanto para a mente, quanto para o corpo.

A utilização não somente de livros como forma de tratamento, mas também de atividades relacionadas ao texto e à leitura, demonstra criatividade e capacidade dos profissionais de lidar com pessoas, problemas, personalidades e idades diferentes, procurando atender às necessidades e anseios da melhor forma possível. Esse tipo de terapia busca promover a recuperação para as pessoas que estejam passando por dificuldades relacionadas à depressão, doenças físicas e/ou psicológicas e problemas emocionais e/ou comportamentais que requeiram atenção. Esse é um tópico que requer mais atenção da sociedade e das companhias médicas, pois suas vantagens são imensuráveis, podendo combater as mais diversas enfermidades.

Chartier (1999) já dizia em seus trabalhos que cada leitor, em cada uma de suas leituras e em cada circunstância, é singular. Vendo desta maneira, a boa seleção dos materiais para a prática da biblioterapia se mostra ainda mais importante, pois é necessário que os materiais de leitura escolhidos realmente alcancem o paciente de uma forma única e diferenciada. Entretanto, seria necessário um conhecimento maior por parte dos bibliotecários e competências na área de psicologia para que as pesquisas fossem expandidas a outras áreas de saúde e de recuperação psíquica.

Sendo a leitura o instrumento primordial da biblioterapia, pode-se afirmar que sem a seleção dos materiais corretos a terapia com leitura não seria possível. É importante ressaltar, conforme já exposto em nossa fundamentação teórica, que a biblioterapia não é uma cura, mas sim um caminho para apaziguar problemas que atormentam as pessoas, ou mesmo uma prática que pode complementar e/ou acelerar o processo de cura. Como a finalidade dessa pesquisa foi procurar por trabalhos que se diferenciassem e mostrassem lados inovadores da utilização da biblioterapia, foi possível observar as práticas, e as enfermidades correspondentes, mais utilizadas pelo mundo afora.

Dessa maneira, a pesquisa pôde verificar quais são os trabalhos biblioterapêuticos realizados e o seu porquê. A leitura dirigida na área terapêutica pode auxiliar a recuperação de crianças, adolescentes, adultos e idosos; e a ampla área de abordagem da biblioterapia atinge doenças completamente diferentes. Assim, conseguir identificar o problema do paciente e relacioná-lo a uma leitura adequada a cada pessoa que se encontra isolada ou hospitalizada, tendo a habilidade de realizar todas as técnicas, pode causar impacto e revelar ações e mudanças positivas na vida dos pacientes.

Como já foi dito, esses projetos são responsáveis por inúmeros benefícios físicos e mentais, pois ajudam a controlar uma série de problemas do paciente/leitor. Sua habilidade em cuidar de emoções, a capacidade 
de transformar os pensamentos, o estudo aprofundado e o prazer desenvolvido pela leitura fazem da biblioterapia uma prática grandiosa.

Diante disso, entende-se a leitura, especialmente a terapêutica, não apenas como decifração de um assunto, algo neutro, mas também, como uma forma de ver o mundo. Focalizando no bem-estar e na reintegração dos pacientes, a biblioterapia pretende ajudar seus usuários a encontrar sentido em suas vidas e, principalmente, revitalizar os modos de pensar, agir, ver o mundo, bem como interpretá-lo.

Vejamos abaixo o levantamento de dados a respeito das iniciativas nacionais que se destacaram ao promover a biblioterapia como instrumento de auxílio para a recuperação de pacientes. Vale ressaltar que os títulos apresentados nas tabelas apenas ilustram o título, não sendo considerado como referência para esse trabalho.

\section{Quadro 1 - Experiências com biblioterapia no Brasil}

\begin{tabular}{|c|c|c|}
\hline Título & Ano & Material \\
\hline Leitura para enfermos: uma experiência em um hospital psiquiátrico & 1987 & dissertação \\
\hline Biblioterapia: uma experiência com pacientes internados em clínica médica & 2000 & dissertação \\
\hline Biblioterapia: uma aplicação na recreação do Hospital de Clínicas de Porto Alegre & 2002 & TCC \\
\hline Biblioterapia para crianças internadas no hospital universitário da UFSC: uma experiência & 2002 & artigo \\
\hline A aplicação da biblioterapia em crianças & 2002 & artigo \\
\hline $\begin{array}{l}\text { Biblioterapia: uma proposta para adolescentes internados em enfermarias de hospitais } \\
\text { públicos }\end{array}$ & 2006 & artigo \\
\hline $\begin{array}{l}\text { A mediação da leitura como recurso de comunicação à criança hospitalizada: subsídios } \\
\text { para a humanização da assistência de enfermagem }\end{array}$ & 2009 & artigo \\
\hline Biblioterapia: uma experiência de ler e contar histórias para pessoas hospitalizadas & 2011 & artigo \\
\hline $\begin{array}{l}\text { Biblioterapia: um estudo de caso da prática de leitura realizada com pessoas com } \\
\text { necessidades psicossociais }\end{array}$ & 2012 & TCC \\
\hline Biblioterapia com Crianças com Câncer & 2012 & artigo \\
\hline Biblioterapia processo de interação que restitui a vida: ler faz bem aos olhos e ao coração & 2013 & TCC \\
\hline $\begin{array}{l}\text { Uso da biblioterapia em pacientes de pediatria: o caso do Projeto Biblioteca Viva em } \\
\text { Hospitais. }\end{array}$ & 2013 & TCC \\
\hline $\begin{array}{l}\text { A contação de histórias para crianças hospitalizadas no Hospital da Criança Conceição } \\
\text { (HCC) }\end{array}$ & 2015 & artigo \\
\hline $\begin{array}{l}\text { Percepções acerca da espiritualidade articulada à biblioterapia enquanto experiência } \\
\text { vivenciada no cuidado integral aos pacientes com HIV e AIDS: uma perspectiva } \\
\text { fenomenológica }\end{array}$ & 2015 & dissertação \\
\hline Biblioterapia no âmbito hospitalar & 2016 & artigo \\
\hline Grupo contadores de histórias: biblioterapia com amor & 2017 & artigo \\
\hline $\begin{array}{l}\text { Implantação de um programa de biblioterapia na Universidade Federal da Paraíba: relatos } \\
\text { de um projeto de extensão }\end{array}$ & 2017 & artigo \\
\hline Mediação de leitura para bebês e crianças: uma experiência no hospital Fernandes Figueira & 2018 & TCC \\
\hline Contação de Histórias em Unidade Oncológica Pediátrica & 2018 & artigo \\
\hline Biblioterapia e a face terapêutica do bibliotecário & 2019 & artigo \\
\hline Biblioterapia - humanização do espaço hospitalar na internação pediátrica & 2019 & artigo \\
\hline $\begin{array}{l}\text { A biblioterapia como fonte medicinal: um estudo sobre a prática da leitura no Hospital } \\
\text { Infantil Varela Santiago - Natal/RN }\end{array}$ & 2020 & TCC \\
\hline
\end{tabular}

Fonte: elaboração dos autores. 
Esse quadro representa a recuperação de 22 artigos entre 1987 e 2020 acerca das experiências com a biblioterapia no Brasil. Por se tratar de uma pesquisa exaustiva, no caso brasileiro, observamos que tais experiências, especialmente as documentadas por meio de artigos ou outros trabalhos científicos, são bem recentes no Brasil. No tópico seguinte apresentamos uma análise mais profunda dessa percepção.

\section{A REALIDADE DA BIBLIOTERAPIA NO BRASIL E NO EXTERIOR}

O levantamento realizado permite reafirmar o potencial da leitura como instrumento terapêutico em ambas as realidades. Quando começamos a pesquisa, a ideia era que nacionalmente teríamos uma menor quantidade de experiências, visto que os estudos sobre o tema são mais recentes e menos abrangentes. Porém, como podemos observar no quadro da seção anterior, a biblioterapia em nosso país tem encontrado tímido apoio, principalmente em projetos hospitalares.

Vejamos que a maioria do material recuperado data dos últimos 10 anos, o que demonstra a visibilidade que ela vem ganhando principalmente na área acadêmica. Nesse sentido, ressaltamos aqui os estudos de Caldin na UFSC.

Quando buscamos pelas palavras-chave 'biblioterapia' nas bases de dados nacionais, nos deparamos com um número razoável de estudos, muitos vindos de universidades do sul do país. Reforçamos que o objetivo do trabalho centrou-se na perspectiva de 'experiências' com a biblioterapia, por isso, mesmo que as pesquisas apresentassem grande quantidade de resultados, quando selecionamos os projetos em si, esse número ficou reduzido, o que não minimiza a ascensão do tema no cenário nacional.

Uma vez voltada ao ambiente hospitalar, essa terapia pode proporcionar uma gama de benefícios aos pacientes. Nesse meio é necessário permanecer sempre atentos, pois a rotina médica, segundo as realidades pesquisadas, torna-se exaustiva. De acordo com Ratton (1975 apud BUENO; CALDIN, 2005, p. 2), "a ampliação do ambiente e a possibilidade de experimentar sentimentos e emoções em completa segurança são os maiores benefícios proporcionados às crianças pelo livro".

O indivíduo ao se deparar com às fragilidades e ao medo decorrente de estar em leitos de hospitais eleva o sentimento de desmotivação por não estar ao lado de seus familiares, entre outros fatores. Sendo assim, não deixar que o dia a dia do paciente se torne vazio é de extrema importância no processo de recuperação física e mental e é por isso que utilizar a biblioterapia e as interações que ela proporciona trazem a sensação de alívio, pois ativam a criatividade e a imaginação e fazem-nas sentirem libertas ao exteriorizarem seus medos; levando-as a uma interpretação da história que vivem pela história que leem, ajudam a manter a mente ativa e apta a desenvolver o senso crítico e ajuda a pensar melhor em suas ações e a manterem-se emocionalmente fortes e preparando-as para a vida, isso sem contar os benefícios básicos que a leitura traz, como o prazer e alegria que são atingidos também por meio dela.

A biblioterapia é indicada, sobretudo, àqueles que necessitem permanecer afastados de seu ambiente familiar, seja em clínicas de reabilitação, hospitais, prisões, ou até mesmo àqueles que procuram fugir de sua realidade por medo de enfrentá-la. Inclui também jovens e adultos que estão vivendo esse mundo de modificações latentes, com tecnologias a todo vapor, pressão por crescimento pessoal e com relações cada vez mais superficiais, fatores esses que desestabilizam o emocional. Neste sentido, a biblioterapia se destaca como instrumento de alívio, conforto para o leitor, e também como um recurso a ser cada vez mais desenvolvido para a construção do caráter e o desenvolvimento de valores culturais e sociais.

O trabalho apresentado por Oliveira et al. (2011), 'O biblioterapêuta: perfil do profissional bibliotecário', mostra um lado do consciente social do profissional, buscando avaliar e estudar a carga de preocupação social que o bibliotecário tende a realizar. É também um modo científico de ver a biblioterapia como uma 'ajuda' que vem de dentro para fora. Nesse texto, o bibliotecário vê a biblioterapia como uma forma de 
recuperação que vem do interior do indivíduo e se expande para ações do cotidiano e no modo de pensar e reagir a situações do dia a dia.

É preciso lembrar que, ao ler um texto, o indivíduo constrói em si outra história ligada às suas experiências pessoais. Assim, a possibilidade de terapia por meio da leitura torna-se real, e admitir esse fato se coloca como ponto de partida no processo de aceitação da condição atual do próprio paciente admitindo que o auxílio por meio da leitura seja uma alternativa válida e divergente.

Visto o cenário brasileiro, vejamos abaixo as experiências de biblioterapia no exterior, recuperadas pela base de dados LISA, no período entre 1980-2020.

\section{Quadro 2 - Experiências com biblioterapia no exterior}

(continua)

\begin{tabular}{|c|c|}
\hline Título & Ano \\
\hline $\begin{array}{l}\text { Bibliotherapie im Rahmen der Psychotherapie bei Patienten mit funktionell-neurotischen Storungen. } \\
\text { Bibliotherapy in the context of psychotherapy for patients with nervous disorders }\end{array}$ & 1980 \\
\hline The myth of bibliotherapy & 1980 \\
\hline Bibliotherapy: a librarian's concern & 1980 \\
\hline Bibliotherapy and the disabled & 1980 \\
\hline Trendy modernej biblioterapie. Trends in modern bibliotherapy & 1981 \\
\hline Bibliotherapy in practice & 1982 \\
\hline Bibliotherapy: its potential in Kenya prisons & 1983 \\
\hline Bibliotherapy in Finnish hospital libraries & 1984 \\
\hline Biblioterapia a neuroticky pacient. Bibliotherapy and neurotic patients & 1984 \\
\hline Can bibliotherapy go public? & 1985 \\
\hline Talking it out: the use of books in discussion for developmental bibliotherapy with children & 1985 \\
\hline Books can help heal! Innovative techniques of bibliotherapy & 1986 \\
\hline Biblioterapia w praktyce radzieckiej. Bibliotherapy as practised in the Soviet Union & 1987 \\
\hline Biblioterapia a konyvtarosi munkaban. Bibliotherapy in library work & 1987 \\
\hline Biblioterapiya v meditsinskikh bibliotekakh. Bibliotherapy in medical libraries & 1987 \\
\hline Biblioterapie met kinders uit enkelouergesinne. Bibliotherapy with children from single-parent families & 1988 \\
\hline Bibliotherapy: practical applications with disabled individuals & 1989 \\
\hline Bibliotherapy and the community library & 1991 \\
\hline The role of bibliotherapy in adjustive behaviour of the handicapped in a developing country & 1993 \\
\hline Bibliotherapy for kids & 1997 \\
\hline Bibliotherapy: Battling Depression & 1998 \\
\hline $\begin{array}{l}\text { Une pionniere de la bibliotherapie: Sadie Peterson Delaney. A pioneer of bibliotherapy: Sadie Peterson } \\
\text { Delaney }\end{array}$ & 1998 \\
\hline Bibliotherapy in school libraries: An Israeli experiment & 2000 \\
\hline The RAYS: Reading and You Scheme: bibliotherapy & 2002 \\
\hline Bibliotherapy: using books to help bereaved children & 2004 \\
\hline FICTION OR NONFICTION? Bibliotherapy Examined & 2005 \\
\hline A study of bibliotherapy service for children in Taiwan's public libraries & 2005 \\
\hline $\begin{array}{l}\text { Biblioterapijos, skaitymo ir gyvenimo kokybes sasajos: tarpdisciplininis poziris. [Bibliotherapy, reading } \\
\text { and life quality coherence: interdisciplinary point.] }\end{array}$ & 2005 \\
\hline The bibliotherapy education project: a collaborative teaching effort goes to the web & 2005 \\
\hline A closer look at bibliotherapy. & 2006 \\
\hline The Reading Remedy: Bibliotherapy in Practice & 2008 \\
\hline Bibliotherapy for Mental Health Service Users Part 1: A Systematic Review & 2008 \\
\hline
\end{tabular}


Reciis - Revista Eletrônica de Comunicação, Informação \& Inovação em Saúde, Rio de Janeiro, v. 15, n. 3, p. 762-775, jul.-set. 2021 [www.reciis.icict.fiocruz.br] e-ISSN 1981-6278

(continuação)

\begin{tabular}{|c|c|}
\hline Título & Ano \\
\hline Medicine for the soul: bibliotherapy & 2008 \\
\hline Bibliotherapy for health and wellbeing: an effective investment & 2008 \\
\hline Helping Children Cope: What Is Bibliotherapy? & 2008 \\
\hline The Bibliotherapy Education Project: Alive and well-and perpetually "under construction" & 2008 \\
\hline $\begin{array}{l}\text { "Computer is not the real world." A screenplay of activities comprising elements of bibliotherapy for } \\
\text { computer-based instruction in the fifth grade }\end{array}$ & 2008 \\
\hline Books on Prescription: Bibliotherapy in the United Kingdom & 2009 \\
\hline Bibliotherapy for Mental Health Service Users Part 2: A Survey of Psychiatric Libraries in the UK & 2009 \\
\hline When I Teach - Bibliotherapy for Children with Intellectual Disabilities & 2009 \\
\hline $\begin{array}{l}\text { When am I afraid of school? Ways to Minimize the Impact of Anxiety and Agression in Children and } \\
\text { Adolescents through Bibliotherapy }\end{array}$ & 2009 \\
\hline Bibliotherapy Program - oswajanie straszakow, Part Two & 2009 \\
\hline $\begin{array}{l}\text { Bibliotherapy Treatment for Children with Adjustment Difficulties: A Comparison of Affective and } \\
\text { Cognitive Bibliotherapy }\end{array}$ & 2010 \\
\hline $\begin{array}{l}\text { The Reading Cure? Bibliotherapy, Healthy Reading Schemes and the Treatment of Mental IIIness in } \\
\text { Ireland }\end{array}$ & 2010 \\
\hline Bibliotherapy in the Work of a Gdynia Library & 2010 \\
\hline $\begin{array}{l}\text { The Role of Bibliotherapy in Value System Formation by Undergraduates in the University of Ibadan, } \\
\text { Nigeria }\end{array}$ & 2011 \\
\hline $\begin{array}{l}\text { The Effectiveness of Acceptance and Commitment Therapy Bibliotherapy for Enhancing the } \\
\text { Psychological Health of Japanese College Students Living Abroad }\end{array}$ & 2011 \\
\hline Reading into Wellbeing: Bibliotherapy, Libraries, Health and Social Connection & 2011 \\
\hline The Bibliotherapy in School Library or Book Discussion & 2011 \\
\hline The Bibliotherapy in School Library or Book Discussion & 2011 \\
\hline A randomized trial of ACT bibliotherapy on the mental health of $K-12$ teachers and staff & 2012 \\
\hline Bibliotherapy for low sexual desire: Evidence for effectiveness. & 2012 \\
\hline Bibliotherapy and Journaling as a Recovery Tool with African Americans with Substance Use Disorders & 2012 \\
\hline Bibliotherapy with children with câncer & 2012 \\
\hline Bibliotherapy for Adopted Children: A Pathway to Healthier Adoptive Identity and Self-Esteem & 2012 \\
\hline Legitimising bibliotherapy: evidence-based discourses in healthcare & 2012 \\
\hline The Implementation of Bibliotherapy in School Libraries & 2013 \\
\hline BIBLIOTHERAPY: a tool for hospital psychologist proceeding in attending the hospitalized child & 2013 \\
\hline Using Bibliotherapy to Enhance Probation and Reduce Recidivism & 2013 \\
\hline Introducing Bibliotherapy in Public Libraries of Jaffna district: an Exploratory Study & 2013 \\
\hline Developmental Bibliotherapy among Juvenile Prisoners & 2013 \\
\hline Mind the Gap: Do Librarians Understand Service User Perspectives on Bibliotherapy? & 2013 \\
\hline Cognitive bibliotherapy and memory training for older adults with depressive symptoms & 2014 \\
\hline $\begin{array}{l}\text { Cognitive-behavioral group depression prevention compared to bibliotherapy and brochure control: } \\
\text { Nonsignificant effects in pilot effectiveness trial with college students }\end{array}$ & 2014 \\
\hline $\begin{array}{l}\text { Indicated cognitive behavioral group depression prevention compared to bibliotherapy and brochure } \\
\text { control: Acute effects of an effectiveness trial with adolescents }\end{array}$ & 2014 \\
\hline Torn out of the family - bibliotherapy to alleviate the trauma of kids living in children's group homes & 2014 \\
\hline Bibliotherapy for young parents - planning and implementing a special interest group & 2014 \\
\hline Bibliotherapy for women & 2015 \\
\hline Bibliotherapy for breast cancer patients after operation & 2016 \\
\hline Bibliotherapy: Its processes and benefits and application in clinical and developmental settings & 2017 \\
\hline
\end{tabular}


(conclusão)

\begin{tabular}{|l|l|}
\hline Título & Ano \\
\hline Bibliotherapy in the age of digitization & 2018 \\
\hline Bibliotherapy at Its Best: Reading Aloud in a Swedish Hospital & 2018 \\
\hline User profile-based bibliotherapy model in academic library & 2018 \\
\hline Innovative service model of bibliotherapy based on participation of medical staff and patients & 2018 \\
\hline $\begin{array}{l}\text { Bibliotherapy-based Wellness Program for Healthcare Providers: Using Books and Reading to Create a } \\
\text { Healthy Workplace }\end{array}$ & 2019 \\
\hline Heroes, Hospitals, [No] Hugs, and Handwashing: Bibliotherapy in the Age of a Pandemic & 2020 \\
\hline Il progetto di bibliotherapy della Biblioteca della Azienda ospedaliera universitaria Federico II di Napoli & 2020 \\
\hline
\end{tabular}

Fonte: elaboração dos autores.

Verificamos, nessa perspectiva, a aplicação da biblioterapia em nichos diferenciados e que limitados a práticas hospitalares. Há trabalhos voltados para educação, para o cenário prisional, jovens vulneráveis e dependentes químicos, entre outros.

Quando a busca foi realizada com a palavra-chave 'bibliotherapy', houve a revocação de aproximadamente 480 materiais. Diante dessa quantidade, fizemos o refinamento para palavras do título com o qual recuperamos 168 artigos. Desses, ao analisar os títulos e resumos em busca dos que privilegiassem as experiências, selecionamos 99. Como no caso brasileiro, os primeiros artigos publicados datam da década de 1980, refinamos para tal época de forma a facilitar a comparação entre as duas experiências, ficando 78 artigos. O termo 'reader therapy' não apresentou resultados.

O que podemos compreender ao analisar a realidade internacional é que os estudos estão bem avançados, principalmente a partir dos anos 2000, e possuem maior interdisciplinaridade. Há um vasto corpo conceitual e de pesquisas para implementação da terapia em diferentes campos. Percebe-se um grande alinhamento com a área escolar e universitária; existe uma preocupação com o emocional dos estudantes, e, também, o interesse em utilizá-la não somente como leitura em si do livro, mas com diversos outros recursos que a biblioterapia permite trabalhar. Outros pontos a serem destacados no parâmetro internacional são os estudos ligados à psicologia comportamental e a preocupação com os jovens.

Sendo assim, estudando os modos de aplicação da biblioterapia, percebe-se o quanto existem resultados satisfatórios. No Brasil, mesmo que seja mais recente a sua utilização, podemos avistar uma ascendência em seus estudos e aplicação. Talvez o conhecimento acerca dos benefícios deste tipo de terapia ainda não tenha alcançado todo o corpo social e acadêmico da área de biblioteconomia, mas essa é uma realidade que está sendo mudada.

Durante o estudo dos trabalhos descritos, pode-se perceber que existiram casos de negação por parte dos pais dos pacientes, e até mesmo dos próprios pacientes, em relação ao tratamento por meio da biblioterapia. Acredita-se que isso ocorreu porque ainda é necessário criar uma base cultural de gosto pela leitura de maneira ampla na sociedade, de modo que, quando houver necessidade de tratar os usuários/pacientes pela biblioterapia, eles já tenham uma apreciação pelos livros e narrativas.

Outro caso decorrente das pesquisas feitas no Brasil é relacionado ao fato de não trazerem confirmação científica de seu trabalho. Muitos desses artigos discorrem sobre a realização e a programação feita, porém, após a consumação do trabalho, não se encontram, na maioria deles, os resultados obtidos com os pacientes depois do período de aplicação, assim como também o fator limitador das áreas estudadas pelos pesquisadores. Observa-se que, ainda que sejam de grande valor, as pesquisas na área da saúde são basicamente todas sobre câncer. O estudo dessa doença é de suma importância, porém, para que o país se torne desenvolvido e especialista na área biblioterapêutica, é necessário que outros estudos sejam 
conduzidos, podendo assim demonstrar que estudos mentais, estudos com jovens infratores, prevenção de doenças psíquicas, entre outros temas, podem ser abordados e pesquisados.

Um ponto positivo que requer atenção no comparativo nacional é que os trabalhos realizados por profissionais bibliotecários são mais recorrentes no Brasil do que nos trabalhos do exterior. São frequentes as iniciativas por parte de bibliotecários que trabalham em hospitais, ou por aqueles que percebem a importância de uma programação deste cunho para a recuperação de crianças e jovens internados. Observando que os pacientes se encontram impossibilitados de prosseguir com a rotina do dia a dia, estes profissionais se empenham em mostrar um lado criativo e inovador, usando do entretenimento para se manterem felizes e ocupados para uma melhor restauração da saúde. Do mesmo modo, percebe-se que nos projetos realizados no Brasil a programação é variada, indo de teatros, peças, leitura em grupo até a leitura silenciosa particular, enquanto nas pesquisas ao redor do mundo foi possível perceber que a leitura já era praticamente prescrita antes de um contato com os pacientes.

Observa-se que no Brasil é como se fosse preciso começar uma ligação pessoal e mais intimista entre os pacientes e os profissionais encarregados. Já no âmbito internacional, são realizados manuais, 'receitas', para que os interessados possam se inteirar do assunto e se 'automedicar', sem contar com a presença de um profissional encarregado.

Nas experiências internacionais notou-se a presença de variados assuntos, desde tratamentos contra a depressão até aos acompanhamentos psíquicos para pessoas portadoras do vírus HIV e, também, para aqueles que sofrem com algum tipo de preconceito ou bullying. Há presença de uma diversidade de profissionais: de professores de escola do ensino fundamental até terapeutas, psicólogos e psiquiatras se encontram envolvidos em projetos decorrentes da biblioterapia.

As pesquisas procuram não somente tratar os pacientes já acometidos por algum tipo de enfermidade, mas sim, prevenir e estabelecer uma corrente forte e positiva. Em casos exclusivos, o projeto já é preparado para alcançar pessoas com características especificas, antes mesmo de haver uma procura pelos pacientes, ou de serem encontrados nos leitos de um hospital. Essa base preventiva da biblioterapia é de grande importância, pois enaltece o valor real dessa prática. Antes mesmo dos pacientes precisarem de remédios ou acompanhamento médico, a biblioterapia, conforme pontuada nos estudos, consegue alcançar os indivíduos da área de risco e lançar sobre estes leituras de autoajuda que promovem o conhecimento interior e auxiliam no processo de reconhecimento das dificuldades e no modo de solucionar os problemas antes que se transformem em algo sombrio e obscuro. A leitura se transforma no apoio necessário para combater sentimentos ruins que podem levar um indivíduo à ruína.

Por fim, não é possível afirmar qual das realidades é melhor, mas é justificável declarar que ambas tem seus pontos positivos, os quais, reunidos, certamente produzirão benefícios extremamente valiosos.

\section{CONCLUSÃO}

A pesquisa permitiu observar a importância da biblioterapia no cunho social e seus benefícios no auxílio do tratamento e manutenção da saúde. Se for bem conduzida e aplicada corretamente, o uso terapêutico da leitura se torna um importante instrumento de transformação social que, na medida em que alcança o paciente necessitado, abre as portas para a interação com a sociedade, entendimento da cultura e alivia as tensões. O bibliotecário e outros profissionais têm utilizado seus conhecimentos nesse sentido, atuando como biblioterapêutas e ampliando seu campo de trabalho.

É necessário analisar as decorrências culturais que envolvem as práticas de leitura no Brasil e no exterior para que se possa observar corretamente o poder da leitura para as pessoas em diferentes situações. Devido ao desenvolvimento avançado de muitos países, a leitura já teve o seu valor consolidado ali há muito mais tempo do que na realidade brasileira. Porém, independentemente deste quesito, observa-se o interesse 
dos profissionais em tornar a biblioterapia conhecida e exaltada em toda a sociedade, validando-a como instrumento valoroso e de importância na luta constante por melhores condições de vida para pessoas com problemas de saúde.

A partir desses aspectos levantados, percebe-se que a leitura se transformou em um apoio sólido e conforto a pacientes que procuram ajuda no processo de enfrentamento e resolução de seus problemas, uma prática válida que já alcançou muitas pessoas. Diante disso, é notável que a biblioterapia tenha sido utilizada em diferentes centros de recuperação clínicos e para a assessoria ao tratamento de doenças físicas, mentais e, também, aos de cunho social no Brasil e no exterior.

Mostrando que seu valor ultrapassa uma mera leitura, as práticas biblioterapêuticas demonstram que as iniciativas na área da saúde psíquica e oncológica no exterior e no Brasil, respectivamente, procuram ajudar pacientes que sofrem por não conseguirem lidar diretamente com seus problemas a encontrarem uma saída para se livrar de seus temores e, ao mesmo tempo, elevar a autoestima e a qualidade de sua saúde.

Ainda que as práticas de biblioterapia não sejam tão utilizadas quanto deveriam nacionalmente em comparação a outros países do mundo, é clara a visão que o Brasil se encontra na direção correta, procurando investir alguns recursos, planejando ações e aos poucos instruindo as pessoas sobre as vantagens adquiridas com a prática biblioterapêutica. Desta maneira, pode-se afirmar que pressupostos deste trabalho foram confirmados, determinando que as práticas de biblioterapia ainda não são utilizadas tanto quanto deveriam no Brasil em comparação com outros países do mundo e que, de fato, a biblioterapia pode ser uma prática que agrega valor na recuperação dos pacientes e que mesmo havendo diferenças entre as práticas de biblioterapia no Brasil e nas práticas realizadas ao redor do mundo, seus benefícios são válidos.

Pode-se concluir que a biblioterapia é uma prática que agrega valor na recuperação dos pacientes, auxiliando no processo de retorno à vida normal. Porém não se pode dizer que a biblioterapia seja a solução ou a cura para todos os problemas existentes, mas esta é um meio alternativo de compreensão de si mesmo.

\section{REFERÊNCIAS}

ALVES, Maria Helena Hees. A aplicação da biblioterapia no processo de reintegração social. Revista Brasileira de Biblioteconomia e Documentação, São Paulo, v.15, n. 1-2, p. 54-61, jan.-jun. 1982. Disponível em: https://www.brapci.inf.br/ repositorio/2011/08/pdf_09e78c51e2_0018372.pdf. Acesso em: 12 fev. 2021.

BUENO, Silvana Beatriz; CALDIN, Clarice Fortkamp. A aplicação da biblioterapia em crianças enfermas. Revista ACB, São José, v. 7, n. 1, p. 157-170, 2002. Disponível em: https://revista.acbsc.org.br/ racb/article/view/372. Acesso em: 13 fev. 2021.

CALDIN, Clarice Fortkamp. A leitura como função terapêutica: biblioterapia. Encontros Bibli: revista eletrônica de biblioteconomia e ciência da informação, Florianópolis, v. 6, n. 12, p. 32-44, 2001. DOI: https://doi.org/10.5007/1518-2924.2001v6n12p32. Disponível em: https://periodicos.ufsc.br/index.php/eb/ article/view/1518-2924.2001v6n12p32. Acesso em: 13 fev. 2021.

CHARTIER, Roger. A aventura do livro: do leitor ao navegador. São Paulo: Editora Unesp, 1999.

FERREIRA, Danielle Thiago. Biblioterapia: uma prática para o desenvolvimento pessoal. ETD - Educação Temática Digital, Campinas, v. 4, n. 2, p. 35-47, 2003. DOI: https://doi.org/10.20396/etd.v4i2.620. Disponível em: https://periodicos.sbu.unicamp.br/ojs/index.php/etd/article/view/620. Acesso em: 13 fev. 2021.

FREITAS, Josilene Santos et al. Biblioterapia: uma experiência com os idosos do abrigo São Francisco de Assis. In: ENCONTRO REGIONAL DOS ESTUDANTES DE BIBLIOTECONOMIA, DOCUMENTAÇÃO, CIÊNCIA E GESTÃO DA INFORMAÇÃO - EREBD, 17., 2014, Fortaleza. Anais [...]. Fortaleza: UFC, 2014. p. 1-11. Disponível em: https://docplayer.com.br/2855427-Biblioterapia-uma-experiencia-com-os-idosos-doabrigo-sao-francisco-de-assis.html. Acesso em: 13 fev. 2021.

GONSALVES, Elisa Pereira. Conversas sobre iniciação à pesquisa científica. 4 ed. Campinas: Alínea; 2007. 
MARTINS, Gilberto de Andrade; LINTZ, Alexandre. Guia para elaboração de Monografias e Trabalhos de Conclusão de Curso. São Paulo: Atlas, 2000.

OLIVEIRA, Ageísa et al. O biblioterapêuta: a nova atuação do profissional bibliotecário. In: ENCONTRO REGIONAL DOS ESTUDANTES DE BIBLIOTECONOMIA, DOCUMENTAÇÃO, CIÊNCIA E GESTÃO DA INFORMAÇÃO - EREBD, 14., 2011, São Luís. Anais [...]. São Luís: UFMA, 2011. p. 1-12. Disponível em: https://docplayer.com.br/71701816-O-biblioterapeuta-a-nova-atuacao-do-profissional-bibliotecario-1. html. Acesso em: 13 fev. 2021.

OUAKNIN, Marc-Alain. Biblioterapia. São Paulo: Edições Loyola, 1996.

RIBEIRO, Gizele Rocha. Biblioterapia: uma proposta para adolescentes internados em enfermarias de hospitais públicos. RDBCl: Revista Digital de Biblioteconomia e Ciência da Informação, Campinas, v. 4, n. 1, p. 112-126, 2006. DOI: https://doi.org/10.20396/rdbci.v3i2.2048. Disponível em: https://periodicos. sbu.unicamp.br/ojs/index.php/rdbci/article/view/2048. Acesso em: 13 fev. 2021.

RODRÍGUEZ-MARTÍN, Boris C.; GÓMEZ-QUINTANA, Anisleidy; DÍAZ-MARTÍNEZ, Glendy; MOLERIOPÉREZ, Osana. Bibliotherapy and food cravings control. Appetite, [s. I.], v. 65, p. 90-95, jun. 2013. DOI: http://dx.doi.org/10.1016/j.appet.2013.02.006. Disponível em: https://www.sciencedirect.com/science/ article/abs/pii/S0195666313000603. Acesso em: 12 fev. 2021. 